\title{
TV/Series
}

$2 \mid 2012$

Les séries télévisées dans le monde : Échanges,

déplacements et transpositions

\section{La représentation de l'espace américain dans The West Wing}

\section{Eric Gatefin}

\section{(Q) OpenEdition}

\section{Journals}

Édition électronique

URL : http://journals.openedition.org/tvseries/1396

DOI : 10.4000/tvseries. 1396

ISSN : 2266-0909

Éditeur

GRIC - Groupe de recherche Identités et Cultures

Référence électronique

Eric Gatefin, "La représentation de l'espace américain dans The West Wing », TV/Series [En ligne],

2 | 2012, mis en ligne le 01 novembre 2012, consulté le 05 mai 2019. URL : http://

journals.openedition.org/tvseries/1396 ; DOI : 10.4000/tvseries.1396

\section{(c) (i) (9)}

TV/Series est mis à disposition selon les termes de la licence Creative Commons Attribution - Pas d'Utilisation Commerciale - Pas de Modification 4.0 International. 


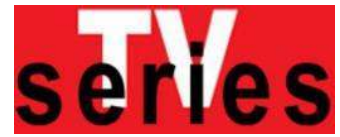

\section{La représentation de l'espace américain dans The West Wing}

Série de couloirs et de déambulations, The West Wing utilise les longs plans séquences au milieu des bureaux pour favoriser l'immersion des téléspectateurs dans un univers très singulier et rendre compte du caractère accaparant du lieu et du travail qui s'y effectue. Devant la prépondérance de cette représentation de l'espace, on peut alors s'interroger : existe-t-il un « ailleurs » de la Maison Blanche dans The West Wing ? La question de savoir ce que l'on voit des États-Unis vaut d'être posée pour une série politique, dont on a souvent loué le caractère pédagogique, c'est-à-dire la capacité à mettre en scène la complexité des problèmes de la société américaine et des questions internationales. Or, si la série présente bien les tenants et les aboutissants des problématiques auxquelles sont confrontés notamment les citoyens américains, quelle représentation propose-t-elle de ces problématiques mêmes ? Que se passe-t-il quand les figures principales de The West Wing délaissent un instant statistiques, clichés discursifs et images télévisées pour entrer en contact avec le pays qu'elles dirigent? Pour les personnages, comme pour les principaux concepteurs de la série, cette rencontre avec l'Amérique est un exercice périlleux où la fiction, l'image du pays construite dans les dialogues à bâtons rompus autour du bureau ovale, heurte quelque chose qui lui résiste forcément et se laisse difficilement réduire.

érie de couloirs et de déambulations, The West Wing (NBC, 19992006) s'inscrit dans la lignée d'E.R. ${ }^{1}$ (NBC, 1994-2009), dans sa manière de traiter l'espace, ou plus précisément de l'utiliser pour filmer le dialogue autrement que par le biais du champ /contre-champ traditionnel. Les longs plans séquences au milieu des bureaux favorisent l'immersion des téléspectateurs dans un univers très singulier, celui de l'aile ouest de la Maison Blanche, où travaille le staff démocrate du président Jed Bartlet. Ces plans ont aussi pour fonction de rendre compte du caractère accaparant du lieu et du travail qui s'y effectue.

Devant la prépondérance de cette représentation de l'espace, on peut alors s'interroger : existe-t-il un « ailleurs » de la Maison Blanche dans The West Wing ? La question de savoir ce que l'on voit des ÉtatsUnis vaut d'être posée pour une série politique, dont on a souvent loué le caractère pédagogique, c'est-à-dire la capacité à mettre en scène de manière claire et dynamique la complexité des problèmes de la société américaine et des questions internationales. Or, si la série présente bien les tenants et les aboutissants des problématiques auxquelles sont confrontés notamment les citoyens américains, quelle représentation propose-t-elle de ces problématiques mêmes ? Que se passe-t-il quand les figures principales de The West Wing délaissent un instant statistiques, clichés discursifs et images télévisées pour entrer en contact avec le pays qu'elles dirigent? Pour elles, comme pour les

\footnotetext{
${ }^{1}$ Rappelons que Laura Innes et Paul McCrane, acteurs récurrents d'E.R. (diffusé en France sous le titre Urgences), ont mis en scène quelques épisodes de The West Wing. Par ailleurs, John Wells fait partie des producteurs principaux des deux séries.
} 
principaux concepteurs de la série ${ }^{2}$, cette rencontre avec l'Amérique est un exercice périlleux où la fiction, l'image du pays construite dans les dialogues à bâtons rompus autour du bureau ovale, heurte quelque chose qui lui résiste forcément et se laisse difficilement réduire.

Trois axes permettraient de rendre compte de ce rapport complexe à l'extérieur qui se noue au gré des sept saisons de The West Wing. Il s'agirait d'abord de noter la prégnance d'une vision inquiète de ce qui se situe hors de la Maison Blanche. Les États-Unis sont d'abord, pour les personnages, un lieu hostile avec lequel leurs rapports ne sont pas aisés et qui favorise donc une attitude de repli.

En marge de cette représentation, les pérégrinations des personnages donnent lieu à des échanges avec des citoyens ordinaires. Deux mouvements sont perceptibles dans les rencontres pittoresques : d'une part, le difficile équilibre à trouver entre l'absence de complaisance et le mépris envers le citoyen ; d'autre part, la tendance idéaliste de la compréhension réciproque entre l'homme ordinaire et l'homme d'État. Il me semble pourtant que la série démontre par ailleurs, et parfois " à son corps défendant », le caractère provisoire ou illusoire de ces moments de grâce, comme si l'espace ouvert vers l'Amérique était immédiatement recadré et que le poids des médiations éloignait immédiatement politique et citoyen. À ce titre, la recomposition de la série autour de la figure du candidat Matt Santos et de sa campagne au cours des Saisons 6 et 7 est symptomatique. Loin de rapprocher candidat et électeurs, la chasse aux voix enclenche un processus où le politique se retrouve à nouveau en vase clos.

La série expérimente la clôture spatiale sous toutes ses formes. Elle la décline à travers ses procédés techniques puisque l'emploi de la steadicam imprime un mouvement qui n'est pas synonyme de liberté, mais donne à voir le confinement des espaces, une structure labyrinthique qui n'aboutit jamais à une ouverture mais à un stationnement ou à une clôture. Fréquemment, la caméra encercle les personnages en tournant autour d'eux. Elle les montre aussi à travers des vitres, l'aile ouest étant à la fois un espace compartimenté et ouvert au regard d'autrui (voir figure 1).

\footnotetext{
${ }^{2}$ Nous n'évoquerons qu'incidemment dans l'article les différences induites par le départ d'Aaron Sorkin, créateur de la série, après la quatrième saison. S'il existe évidemment des évolutions au cours des sept saisons, il faut aussi remarquer que grâce à un grand nombre de réalisateurs et de producteurs fidèles (Alex Graves, Christopher Misiano, Thomas Schlamme, John Wells) l'ensemble de la série est d'une grande homogénéité.
} 


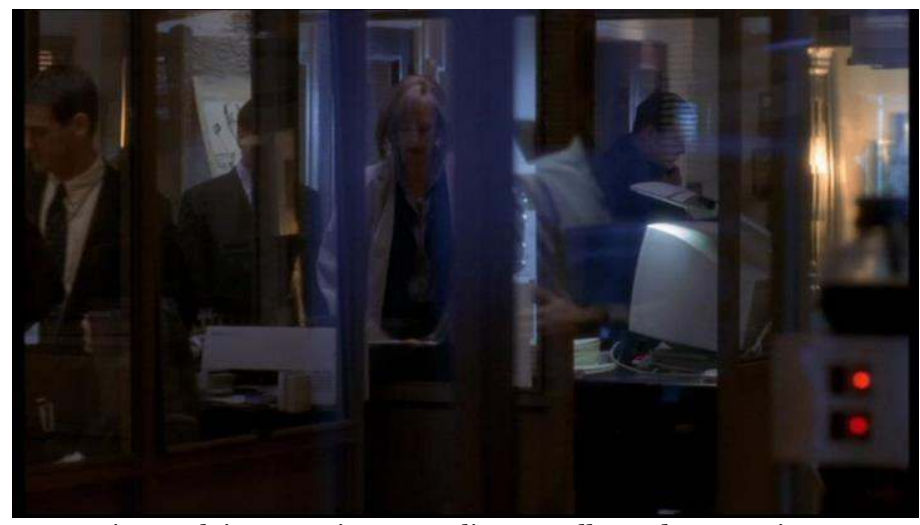

Fig. 1 : Cloisons et vitres - un lieu aux allures de panoptique.

Ces figures répétées rejoignent certains gimmicks de la série : on pense au poisson rouge offert à C.J. Cregg (voir figure 2), officiant elle-même dans une salle de presse qui a toutes les allures d'un lieu où on l'assiège.

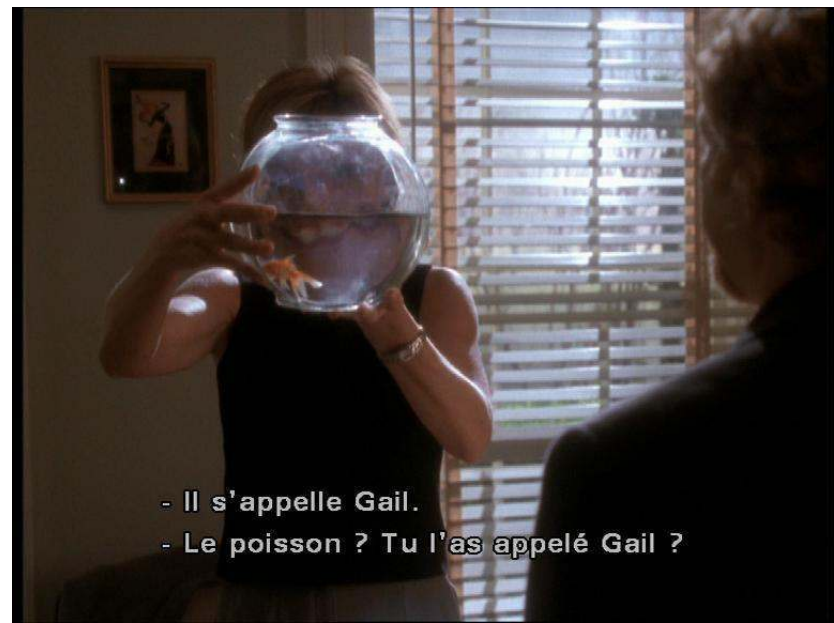

Fig. 2 : C.J. Cregg, requin de la politique ou petit poisson enfermé dans un bocal?

La balle que les conseillers en communication projettent sur la vitre séparant leurs bureaux pour s'interpeller sans se déplacer ni parler crée pour le spectateur un effet de surprise. Elle rappelle surtout avec insistance la présence des cloisons. Enfin, remarquons l'intérêt des scénaristes pour les dispositifs qui enferment des personnages 
pourtant rarement extraits de leurs bureaux. Toby Ziegler se trouve assigné à résidence tout au long de la septième saison, C.J. Cregg est placée sous surveillance et gênée dans ses mouvements (3.20); une suspicion d'attaque est prétexte à la mise en place d'un huis clos (4.20).

De manière emblématique, l'un des épisodes (4.12) qui éloigne radicalement le personnage de C.J. Cregg de l'univers clos de l'aile ouest n'offre que l'apparence d'une pause pour le personnage. Auprès de son père atteint de la maladie d'Alzheimer, la porte-parole de la Maison Blanche retrouve les mêmes phénomènes d'enfermement psychologique, de vaine course contre la montre. Mal à l'aise dans l'espace de sa jeunesse, engoncée dans des cadres qui fonctionnent comme des refuges mais semblent aussi l'étouffer, C.J. aboutit au constat que la maladie dont souffre son père est une figure de ce qu'est l'existence ordinaire, caractérisée par l'amnésie, le chaos et l'absurdité. Si son père paraît prisonnier du mal qui le ronge, sa situation ne diffère guère de celle de sa fille, étrangère à elle-même à force de s'identifier à son rôle. Ainsi, alors que les personnages paraissent déjà happés par leur lieu de travail, les scénaristes trouvent constamment de nouveaux moyens de remettre au premier plan le principe d'enfermement, même à l'extérieur de la Maison Blanche.

Cette clôture, qui a ses connotations douloureuses, s'inscrit dans une logique d'aseptisation de l'existence, prisonnière permanente $\mathrm{du}$ jeu politique. D'existence privée il ne peut y avoir dès lors que l'achat d'un véhicule tout-terrain (6.5), les amours avec une call-girl (1.1) ou les attaques de la maladie (1.12) ont vocation à devenir des faits publics dont il faudra maîtriser les effets dévastateurs. Tout pousse donc à l'endogamie dans cette représentation de l'univers politique. Et les personnages comme les scénaristes s'en convainquent progressivement. Les conseillers se séduisent3, se reconnaissent dans leur singularité, car l'enfermement développe des tics largement repérables: les mots d'esprit, le sens de la dérision ou de l'autodérision, la fatigue et le dévouement; à ces traits s'ajoute le sentiment d'être différent des autres. Ainsi, lors d'une soirée à Los Angeles (1.16), les conseillers du président se sauvent les uns les autres en écartant un interlocuteur perçu comme un étranger, avec lequel on n'a rien à partager. Il n'est pas anodin que le seul personnage avec lequel ils arrivent finalement à s'entendre soit une femme sourde, Joey Lucas, dont le métier est justement de concevoir et d'interpréter des sondages, ce qui fait d'elle la voix de l'opinion publique aux yeux des personnages (voir figure 3).

${ }^{3}$ Dans son intervention au forum des images, Carole Desbarats a souligné l'importance des jeux de séduction au sein de la série, faisant référence à la comédie américaine hollywoodienne des années 1930 ( $" \dot{A}$ la Maison Blanche analysé par Carole Desbarats », cours du 15 avril 2011, www.forumdesimages.fr/fdi/L-Academie/A-voir-a-ecouter/LesCours-de-cinema-en-video, consulté le 5 août 2011). 


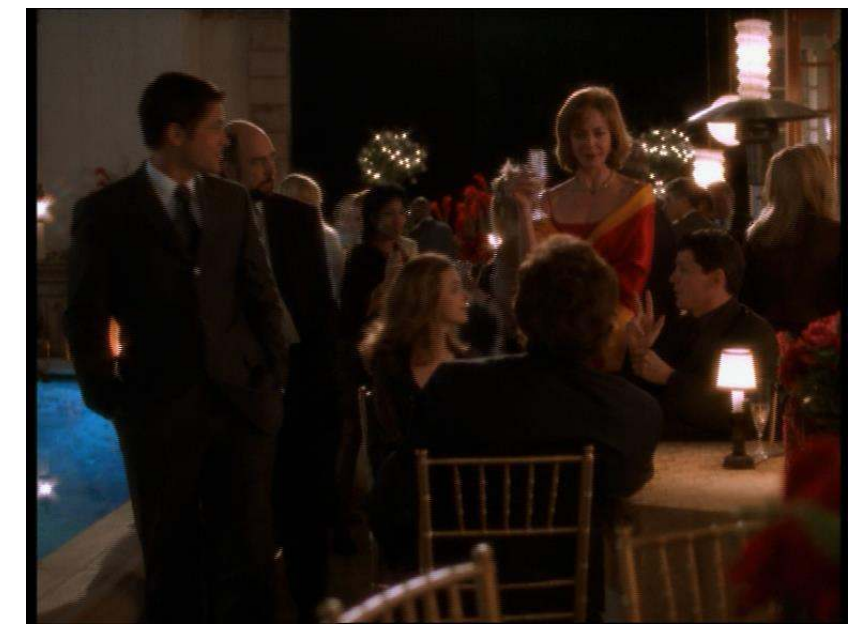

Fig. 3 : Joey Lucas (la jeune femme assise au centre) devient l'objet de l'attention de tous les conseillers du président Bartlet, toujours par l'intermédiaire de son interprète.

L'autre facette de ce repli serait donc, on le voit, un désir de ne pas sortir de sa communauté, l'ailleurs et les autres suscitant souvent l'ennui, l'incompréhension mais parfois plus radicalement l'inquiétude ou la peur. Dans The West Wing, le cliffhanger de fin de saison consiste fréquemment en une sortie publique qui tourne mal (voir figure 4).

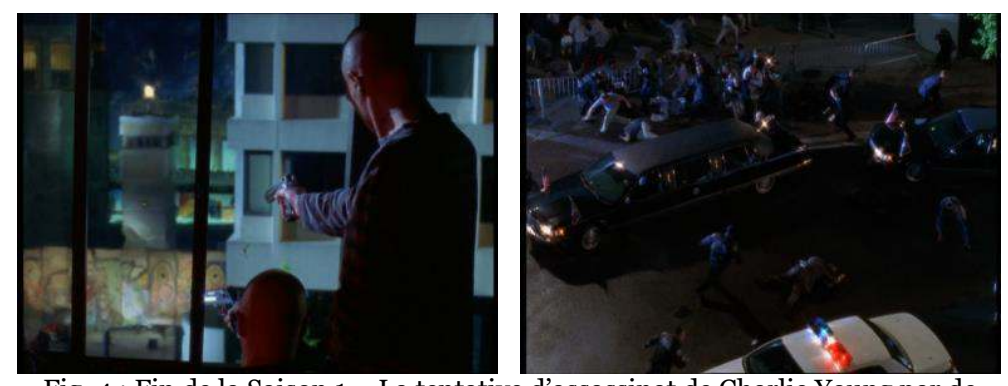

Fig. 4 : Fin de la Saison 1 - La tentative d'assassinat de Charlie Young par de jeunes néo-nazis

Le traumatisme classique de l'attentat politique, figure obsessionnelle de l'histoire des États-Unis et de son cinéma, se décline en enlèvement, fusillade ou attaque à la bombe qui, la plupart du temps, se déroulent sur le sol américain. Dès la fin de la première saison, Aaron Sorkin donne le ton : l'équipe du président est la cible d'un attentat perpétré 
par de jeunes néo-nazis qui ne supportent pas la présence de Charlie Young, un afro-américain, dans le cortège (1.22). Même si les périls extérieurs ont leur place dans la série, l'accent est en effet mis sur la violence que peuvent manifester les Américains contre leurs élites. Il est important cependant de relier ces moments où la crise est rendue nécessaire par le dispositif de la série ${ }^{4}$ avec d'autres épisodes apparemment moins marquants. Ainsi, lors d'une visite dans le comté d'Orange, de tradition très conservatrice (4.16), l'ex-épouse d'un des conseillers est prise à partie par un couple qui s'offusque de voir une femme députée enceinte et divorcée. On reproche également à Charlie Young de s'afficher avec la fille du président. Ce qui est dit à travers cette altercation qui s'achève au poste de police, c'est que l'ennemi est bien souvent intérieur. Sorkin montre d'abord ses héros aux prises avec une Amérique ordinaire qui n'a rien de reluisant. Gangrénée par le racisme, le puritanisme ou par une violence aveugle, la société apparaît sous un jour inquiétant (voir figure 5).

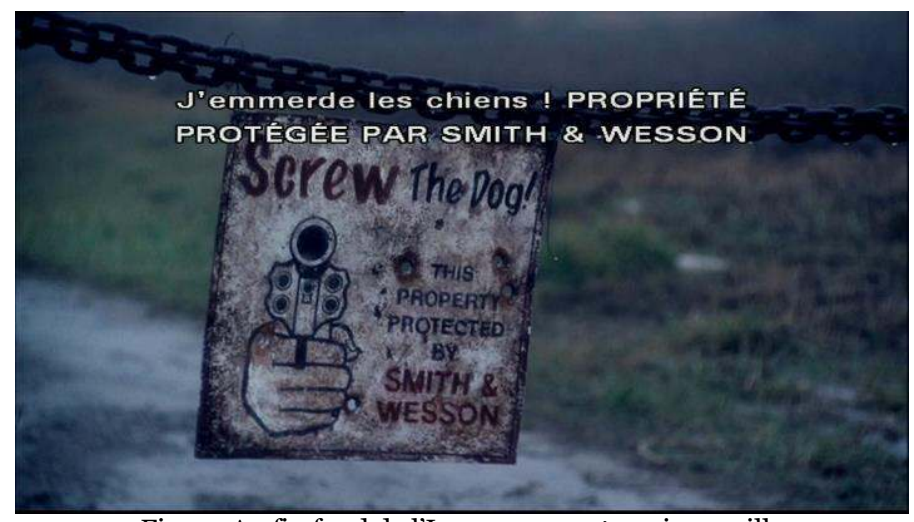

Fig. 5 : Au fin fond de l'Iowa - pancarte qui accueille

les visiteurs d'un potentiel candidat... démocrate !

Cette vision paranoïaque d'une population perçue comme potentiellement dangereuse ne s'impose pas de manière univoque. Elle est présentée comme une tendance presque naturelle dans la mesure où Sorkin et Wells proposent aux téléspectateurs le point de vue d'un groupe qui vit à la marge de l'Amérique ordinaire et n'a l'habitude de la voir que d'une manière médiatisée.

4 On notera à cet égard qu'Aaron Sorkin et John Wells font preuve d'un grand classicisme dans la construction dramatique des fins de saisons. Par ailleurs, le déroulement des épisodes est en général assez conventionnel du point de vue des intrigues. Si des aspects formels ou narratifs singuliers sont à souligner, il faudrait plutôt chercher du côté du traitement du temps. L'usage du flash-back, qui n'est certes pas une nouveauté, nous semble riche d'effets de sens dans de nombreux épisodes. 
La construction narrative des sept saisons, où trois campagnes présidentielles sont dépeintes de manière de plus en plus précise, offre cependant l'occasion d'une rencontre suivant d'autres modalités entre politique et citoyen. Les excursions des conseillers du président ouvrent des perspectives terriblement absentes par ailleurs: quelles sont les préoccupations et problèmes de cette Amérique si diverse ? Quelle est la perception par le citoyen des décisions de ses représentants?

Faisant écho à un épisode de la Saison 1 intitulé « Twenty hours in L.A. » (1.16), l'épisode double "Twenty hours in America » (4.1 et 4.2) met en scène, parmi ses lignes d'intrigue, le périple de deux conseillers, Josh et Toby, et de leur assistante Donna, alors qu'ils tentent de quitter l'Indiana, où ils ont été malencontreusement oubliés par le cortège présidentiel. Pour eux, fréquenter les rednecks est à coup sûr un acte manqué, la marque d'un dysfonctionnement dans leur emploi du temps. Les scénaristes placent les spectateurs entre rire et malaise: les politiciens civilisés affichent mépris, goujaterie et aveuglement à l'égard d'une population qui ne se laisse pas réduire à un stéréotype.

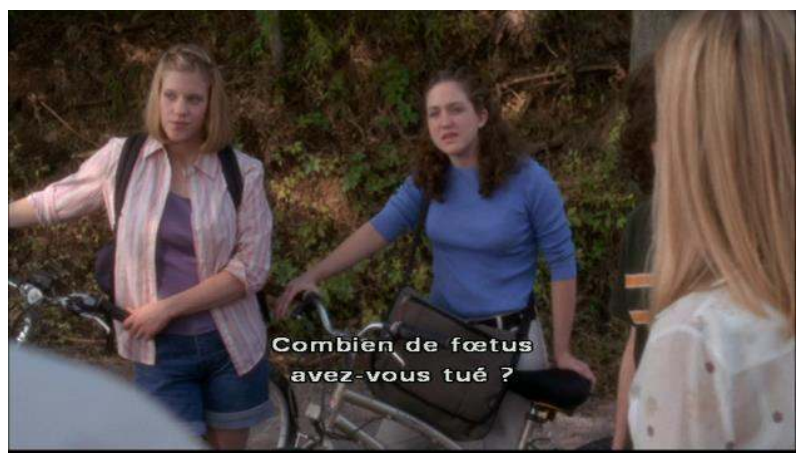

Fig. 6 : Les démocrates en terrain hostile, ou la question de l'avortement comme ligne de partage infrangible.

À côté d’individus farouches qui énoncent sans réserve leur antipathie envers les démocrates, de jeunes filles qui revendiquent leur position anti-avortement (voir figure 6), on trouve une agricultrice diplômée travaillant aussi dans l'assurance ou un jeune homme marginal mais dévoué. Avec toutes ces personnes, Josh et Toby ne savent pas adopter une attitude bienveillante.

Il faudra un drame national, un hôtel et un bar, lieux familiers, pour que les deux hommes donnent enfin un sens à ce contact avec l'Amérique. Le road movie, marqué par des rencontres ratées et des 
détours subis 5 , aboutit enfin à un échange fructueux où Sorkin rapproche idéalement les élites du peuple ${ }^{6}$. Vision idyllique où les différences sont transcendées et où le pas est franchi par le politique qui accepte de se mettre à l'écoute d'un cas singulier (voir figure 7).

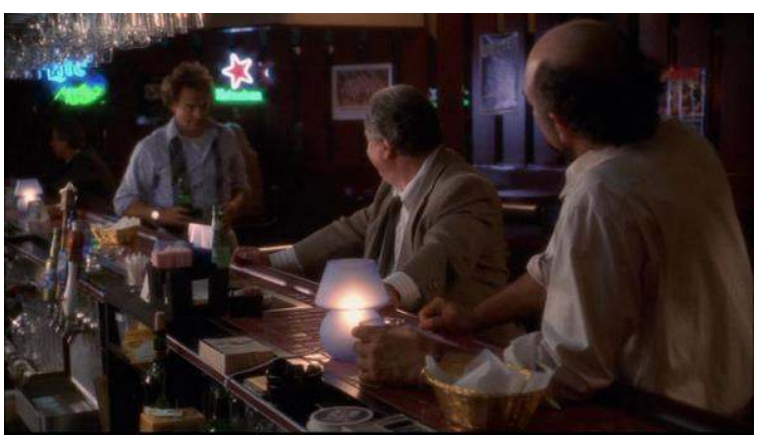

Fig. 7 : Josh Lyman (à gauche, de face) et Toby Ziegler (à droite, de dos) se mettent enfin à l'écoute des difficultés d'un homme ordinaire.

D'évidence, la série opère là à contre-courant de son schéma ordinaire. Inclus dans la fiction ou public extérieur visé par les créateurs de la série, le «common man » est invité à se mettre à la hauteur des enjeux qui font le quotidien des décideurs politiques. Quel que soit le problème évoqué, il s'agit d'en dépeindre la complexité et de montrer le politique en butte à un dilemme. Manière de rendre les spectateurs plus intelligents ou manière de légitimer des choix effectués par des professionnels dotés d'une hauteur de vue hors du

\footnotetext{
5 Depuis les années 1970, la forme du road movie a subi de nombreuses transformations dans la fiction hollywoodienne. Parcours problématique, où la question de la destination n'a rien d'évident, le road movie est paradoxalement prétexte à une réflexion sur le temps. Pour une mise en perspective de ces aspects, on lira avec profit Jean-Baptiste Thoret, $L e$ cinéma américain des années 7o, Paris, Cahiers du Cinéma, 2006.

6 Notons au passage le rôle d'intermédiaire joué par certains personnages de la série. Charlie Young représente les catégories défavorisées et leur ambition de parvenir à un statut élevé dans la société par leurs talents et leur intelligence. Donna Moss, d'abord secrétaire de Josh Lyman, conseiller du président, pose souvent les questions qui seraient celles d'un citoyen ordinaire à propos de problèmes économiques ou politiques. Elle tient une grande place dans le dispositif pédagogique de description des institutions politiques américaines, puisqu'elle fournit l'amorce à des explications destinées aux téléspectateurs. Dans l'épisode «Twenty hours in America », ce personnage sert de relais entre les conseillers du président et les Américains « ordinaires » qu'ils rencontrent. Tout se passe comme si elle savait parler deux langues. Par ailleurs, elle est seule en mesure de gérer les côtés pratiques de ce voyage qui tourne mal ; enfin, c'est elle qui provoquera la remise en question de Josh et de Toby, en soulignant leur mépris à l'égard des citoyens croisés lors de leur périple. Il est très significatif de voir les personnages de Charlie et de Donna évoluer dans les deux dernières saisons vers un plus grand professionnalisme politique, qui les éloigne progressivement de leurs origines. Ils perdent alors le recul qui était le leur et se trouvent pleinement immergés dans un univers dont la logique propre gouverne leurs discours, leurs idées et leurs actes.
} 
commun? Arnold Vinick, républicain face aux agriculteurs (6.13) ou Jed Bartlet, démocrate face à un producteur laitier (2.1) apparaissent héroïques car ils vont à l'encontre d'un accord démagogique avec leur public du moment. La rencontre avec l'Américain ordinaire vise alors à l'élever, à lui faire comprendre d'autres intérêts que les siens en vue d'un bien commun.

Le propos de la série consiste à répéter, épisode après épisode, ce mouvement. Il est aussi de rappeler avec insistance que le politique n'a pas à se préoccuper durablement de situations individuelles, aussi tragiques soient-elles. Quand le président, encore traumatisé par l'enlèvement raté de sa fille, rend visite aux sinistrés d'un cyclone (5.6), son staff n'a de cesse de lui rappeler qu'il faut regagner la Maison Blanche. L'attitude compassionnelle du personnage, l'écoute qu'il prodigue aux citoyens bouleversés, son ravalement au rang de «common $\operatorname{man}^{7}$ » (voir figure 8) deviennent rapidement des éléments problématiques.

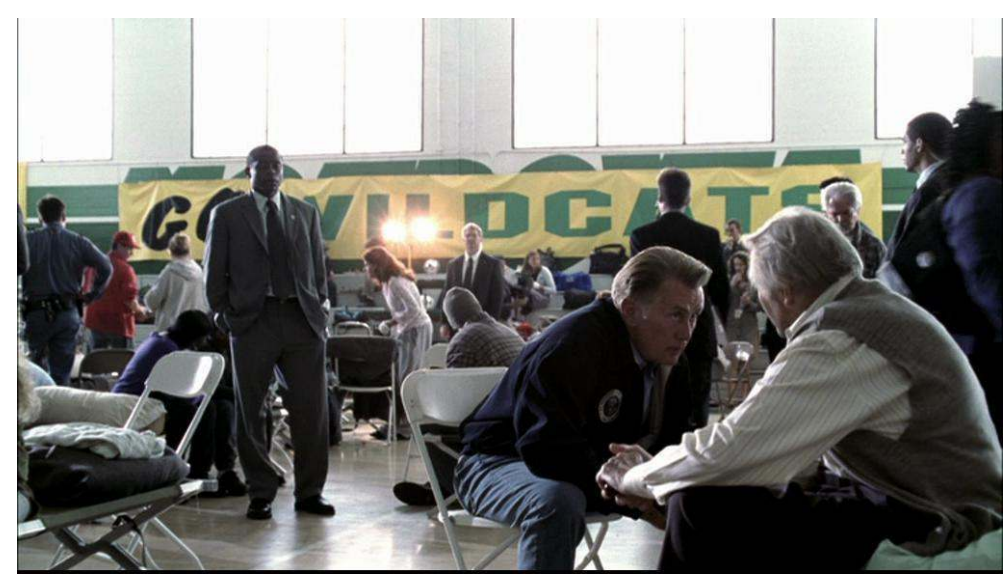

Fig. 8 : Bartlet, assis au centre de l'image,

supprime toute distance avec les gens ordinaires.

Le président perd du temps - il y a comme une vacance du pouvoir en son absence (voir figure 9).

\footnotetext{
${ }_{7}$ Sur l'image du président des États-Unis dans les fictions hollywoodiennes, on consultera avec profit Anne-Marie Bidaud, Hollywood et le rêve américain; cinéma et idéologie, Paris, Masson, 1994.
} 


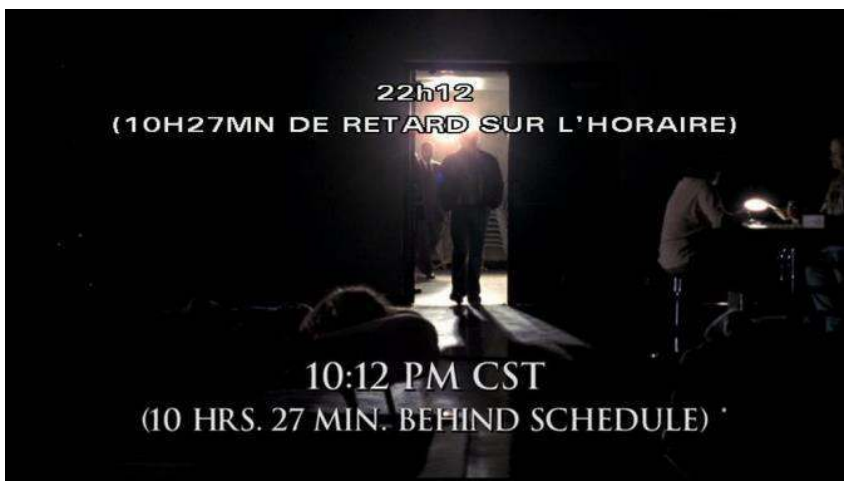

Fig. 9 : L'obsession des horaires et du retard des incrustations qui scandent l'épisode.

L'attention portée aux citoyens perçus comme des individus se transforme en incapacité à diriger la collectivité, en aveu de faiblesse d'un homme rongé par un sentiment de culpabilité. Le discours que lui tient C.J. Cregg, porte-parole de la Maison Blanche, est extrêmement clair : elle attend du président une posture de dirigeant susceptible de rassurer le peuple américain face aux menaces extérieures. Tout se passe comme si, doté de pouvoirs extraordinaires, le «leader du monde libre ${ }^{8} »$ devait renoncer aux contacts ordinaires avec des individus pour agir seulement à grande échelle.

Les gestes que fait le politique pour approcher le citoyen au sein d'un espace caractérisé par la démesure constituent comme des instants volés, dont la légitimité est suspecte. L'accès du personnel de la Maison Blanche aux citoyens ordinaires se révèle complexe ; une fois les représentations des deux camps battues en brèche se pose le problème de l'arbitraire de ces rencontres. Est-il légitime qu'elles influencent la réflexion de politiques sommés de ne raisonner qu'en globalité ? S'il convient donc que le citoyen prenne le temps de comprendre le politique, ce dernier doit à tout moment se recentrer sur l'exercice du pouvoir et a vocation à ne s'adresser qu'à un entourage proche, professionnel et informé9.

8 L'expression est complaisamment employée tout au long de la série par différents personnages. Si le personnage de Bartlet, incarné par Martin Sheen, éprouve parfois l'impression d'être un colosse aux pieds d'argile, il est rare que son entourage fasse preuve d'un grand recul sur la grandeur de la fonction de président des États-Unis.

9 La question de la démagogie est l'une des thématiques centrales pour la compréhension de la série. Elle mérite d'être posée dans cet épisode : C.J. Cregg rappelant le président à ses devoirs tiendrait un discours anti-démagogique dans la mesure où le départ de Bartlet pourra être perçu comme un abandon de la part de la population. Tout est en fait plus complexe : d'abord, la compassion manifestée par le président est évidemment présentée comme parfaitement sincère ; par ailleurs, son souci de constater les dégâts matériels, de rencontrer la population, de discuter avec des individus et de vivre un peu auprès d'eux 
La gestion des espaces dans la série témoigne de ce perpétuel souci de recadrage, qui isole les personnages du monde extérieur. Les politiques semblent absents des lieux qu'ils arpentent. Ces lieux perdent eux-mêmes de leur épaisseur. Purs espaces de passage, les tarmacs semblent en contradiction nette avec les lieux ordinaires de la série, essentiellement exigus. Sans identité et simplement fonctionnels, ils reflètent en fait assez justement le caractère secondaire du décor, sans cesse investi par des corps bavards, souvent agités, qui n'ont cure de ce qui les entoure (voir figure 10).

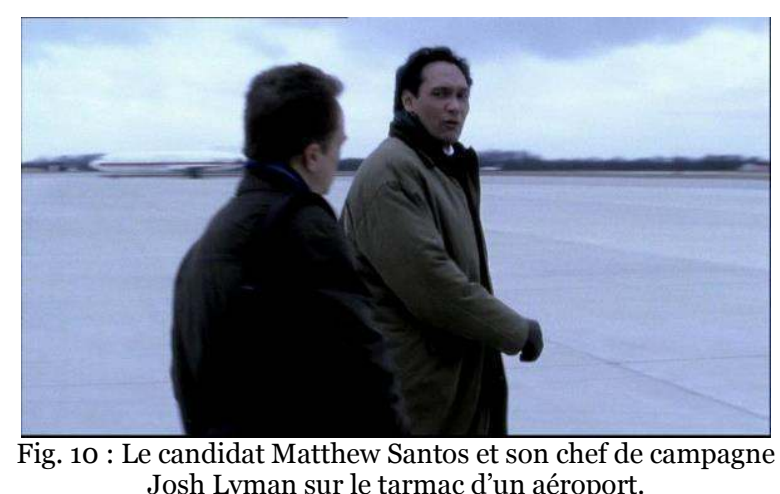

Dans la mécanique des campagnes électorales, les différents États et leurs décors singuliers devraient prendre d'assaut l'écran. Or, de la campagne de Santos pour obtenir l'investiture démocrate à son duel face au républicain Vinick, l'espace se resserre. Producteurs et réalisateurs travaillent, au cours de ces deux dernières saisons, selon deux axes distincts. D’un côté, on observe des expérimentations sur les teintes d'images, les lumières. D'un autre côté, le cadre et l'organisation des lieux sont pensés en termes de continuité. Pour conserver la fidélité de téléspectateurs qui doivent reconnaître immédiatement la série, le traitement du décor vise à retrouver le rythme et la logique de parcours et de station dans les espaces confinés de l'aile ouest.

Un passage de la septième saison révèle bien ces différents aspects $\left(7.13^{10}\right)$. On suit d'abord les personnages de Will et de Kate, deux conseillers proches du président en place, dans les couloirs de l'aile ouest, au cours d'une scène classique dans son découpage et son

témoigne d'une approche où l'émotion et la raison sont également sollicitées ; enfin, la porte-parole de la Maison Blanche délivre un discours où la part de stratégie politique se dissimule sous une argumentation censée emporter l'adhésion des téléspectateurs mais dont les implicites sont moins recommandables qu'il n'y paraît.

${ }^{10}$ Le passage en question se situe dans les dix premières minutes de l'épisode. 
contenu, où l'intime resurgit au cœur du politique. Sur un rythme d'abord similaire puis plus rapide, la caméra accompagne ensuite Matt Santos et son staff dans les coulisses d'un hôtel jusqu'au parking. L'éclairage est plus cru, les déplacements de la caméra et les raccords un peu plus heurtés. Retour enfin à une scène plus proche de la première dans son atmosphère. Après les couloirs des bureaux puis ceux d'un hôtel, la caméra déambule dans l'allée d'un avion où Vinick et ses proches discutent stratégie. Ainsi, les trois scènes juxtaposées fonctionnent globalement sur les mêmes principes. La seconde montre le lieu privilégié des Saisons 6 et 7 : c'est l'hôtel avec ses chambres, ses couloirs, ses ascenseurs aussi, dans lesquels la parole s'interrompt ou peut devenir plus grave, plus sérieuse. Les moyens de transport sont également investis. Bus et avions comportent des cloisons, des couloirs où les mouvements, aussi embarrassés soient-ils, demeurent possibles. Dans The West Wing, en effet, un voyage ne peut jamais être une simple attente, un temps sans discussion où les lieux prendraient le dessus sur les personnages. Les espaces sont là avant tout pour être traversés, anéantis par le mouvement.

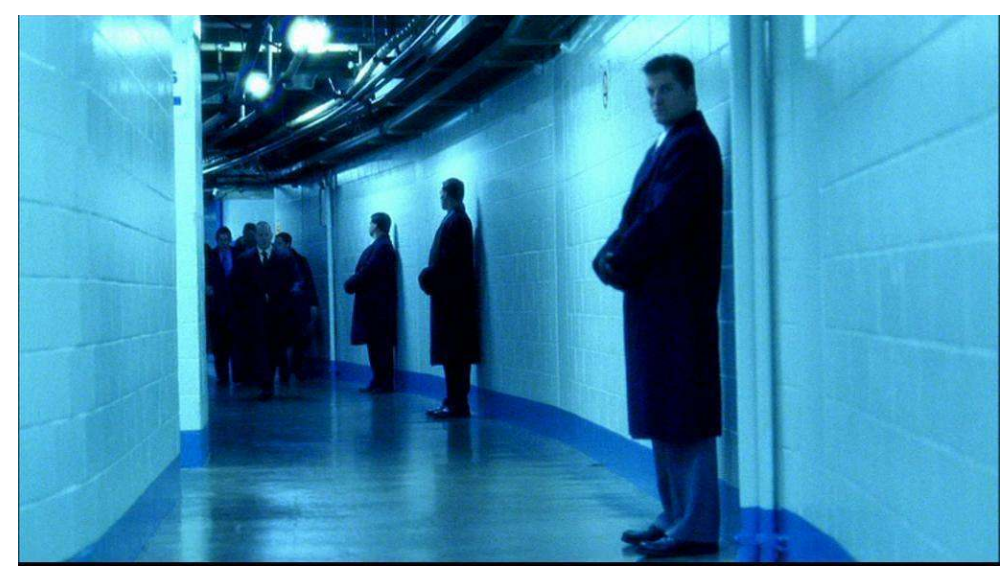

Fig. 11 : Un candidat à l'investiture démocrate dans les coulisses, avant d'entrer en scène pour un discours.

Lieu de la transition, de la répétition et lieu anonyme par excellence, la coulisse est l'objet d'une attention particulière de la part des réalisateurs (voir figure 11). On y retrouve les plans séquences qui donnent à voir les cloisons et les transpercent simultanément. Mais on aperçoit aussi autre chose : un travail sur la lumière, dont les sources sont souvent exhibées crument et constituent l'éclairage principal de la scène (voir figure 12). 

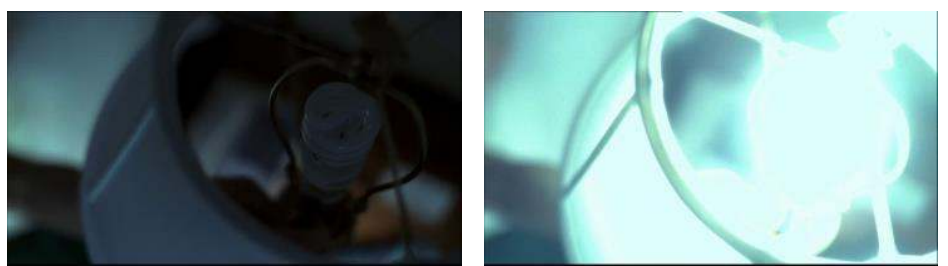

Fig. 12 : Teintes bleutées, entre froideur et incandescence.

Les dominantes de bon nombre d'épisodes de campagne sont les couleurs froides, le bleu, le blanc, accentuées par des lumières surexposées, celles des néons par exemple, rendus extrêmement présents par des contre-plongées systématiques dans les couloirs et les coulisses d'hôtel (voir figure 13).

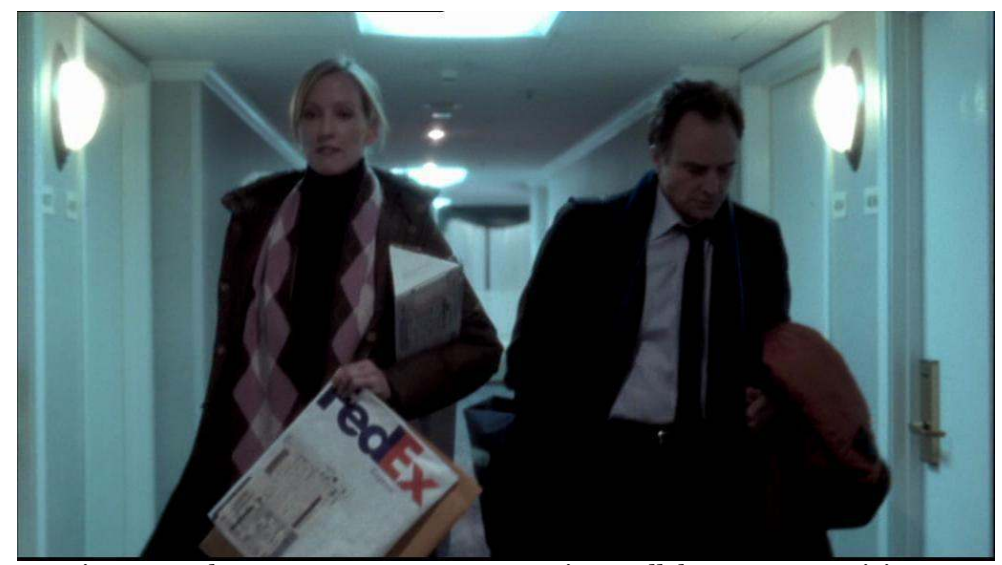

Fig. 13 : Josh Lyman et Donna Moss, anciens collaborateurs, participant désormais à la campagne de candidats adverses.

Plusieurs effets se conjuguent alors: une impression documentaire renforcée par une caméra qui tremble parfois un peu plus dans sa manière d'accompagner les personnages, un effet d'extérieur qui vient contraster avec les teintes de la Maison Blanche où se joue non pas la conquête mais l'exercice du pouvoir, un effet d'aseptisation aussi, qui rappelle constamment le vase clos dans lequel vivent ces personnages, tous baignés dans la même ambiance. Ils sont à la fois extérieurs aux États, qu'ils réduisent aux chiffres de sondages, extérieurs à la politique menée par Bartlet, qui ne les concerne que ponctuellement, extérieurs aussi à eux-mêmes puisque leur vie intime, affective est renvoyée vers les marges et devient moins naturelle que leur quotidien politique. Le cas de l'intrigue sentimentale entre Josh Lyman et sa secrétaire Donna, toujours avortée dans les saisons 
précédentes et finalement entamée dans les ultimes épisodes, est, malgré les apparences, exemplaire de ce bannissement de l'intime. L'idylle entre les deux personnages débute à proprement parler à l'instant même où les sondages donnent enfin Santos vainqueur, à quelques jours du scrutin. À l'attirance sexuelle se mêle alors le désir irrépressible de ne jamais perdre de vue une campagne sur le point d'aboutir. La télévision et tous les outils de communication investissent la chambre à coucher, l'urgent n'étant pas forcément de s'aimer mais plutôt de gagner (7.16).

Derrière l'indéniable beauté plastique du travail sur l'image effectué notamment par Alex Graves, se joue donc peut-être le constant rappel d'une médiation infranchissable entre les élites et leur territoire national. Il faut là rappeler une évidence: pour les conseillers du président, l'Amérique se voit d'abord à l'écran. La plupart du temps, c'est par ce biais qu'ils vont être informés d'événements les détournant de leurs préoccupations du moment. La logique de l'information continue bouleverse en permanence le fonctionnement de l'aile ouest et infléchit notamment le travail de C.J. Cregg, appelée elle-même à intégrer le flot d'images déversées, par l'intermédiaire de ses conférences de presse ${ }^{11}$ (voir figure 14).
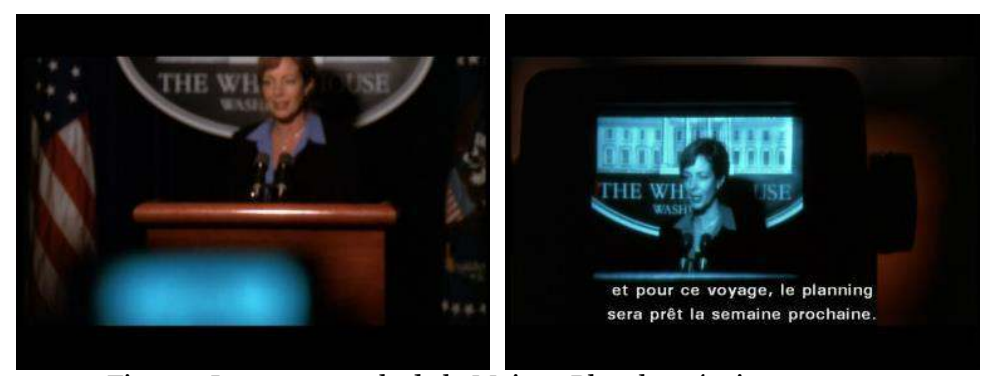

Fig. 14 : La porte-parole de la Maison Blanche, réagissant souvent à des images et devenant l'une de ces images télévisées.

Le paradoxe majeur de la série consiste à déployer pour les téléspectateurs ce qui se déroule dans un microcosme étriqué, celui d'une aile ouest découpée en couloirs, bureaux et salles de réunion, tandis que l'espace américain tient tout entier dans une télévision ou

\footnotetext{
${ }^{11}$ On signalera notamment l'exercice de style consistant à présenter un épisode de la série comme un documentaire suivant une journée de C.J. Cregg. En devenant une figure de reportage télévisé, le personnage devient étonnamment proche des téléspectateurs. Grâce à cet épisode, ces derniers peuvent paradoxalement croire d'autant plus à son existence réelle qu'elle est censée être filmée par des journalistes (5.18).
} 
une carte des États ${ }^{12}$, et le monde même sur les écrans de la salle des crises (voir figure 15).

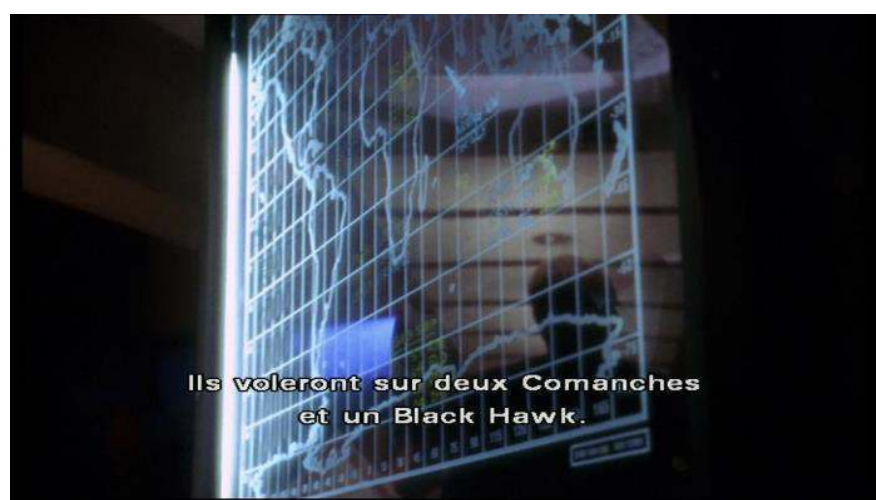

Fig. 15 : Carte et géostratégie dans la salle des crises.

Engoncés dans la petite lucarne, les États-Unis d'Amérique subissent aussi de plein fouet les dérives politiciennes de la campagne électorale. Wells nous montre au fur et à mesure des Saisons 6 et 7 l'engrenage qui enferme le candidat démocrate Santos dans un discours calculé et une logique cynique de conquête de voix. La Saison 6 avait pour but d'opposer, lors des primaires démocrates, un candidat opportuniste, Bob Russell, à un Santos sincère, pétri de convictions et résolu à ne pas transiger sur elles. La Saison 7 montre Santos et son homologue républicain à la chasse aux voix et peu regardants sur les moyens de les obtenir. Arnold Vinick se laisse tenter par l'un de ses conseillers, qui s'est retrouvé en possession de documents compromettants (7.14) appartenant à son adversaire. Matthew Santos exploite quant à lui sans vergogne son image de jeune homme dynamique et séduisant, réserviste dans l'armée de l'air (7.1 et 7.2). Pourtant, jusque dans ces dérives, les scénaristes offrent constamment des contrepoints qui ramènent in fine ces personnages vers la grandeur. Là où le calcul politique dépasse les bornes de la décence, les deux candidats font machine arrière ou se trouvent déchargés de la responsabilité de manœuvres qui sont le fait de leur entourage. Tous deux incarnent jusqu'au bout une vision idéaliste de l'action et de la morale politique (voir figure 16).

12 C'est le cas notamment dans les deux dernières saisons, où les questions électorales donnent à envisager les États-Unis d'après une carte des États et de leur couleur politique. 

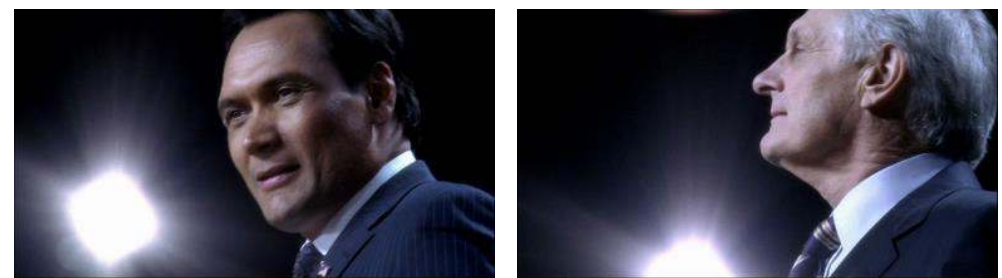

Fig. 16 : Deux figures de la grandeur, éclairées par une lumière qui les magnifie : des candidats démocrate (à gauche) et républicain (à droite) qui incarnent un idéal des États-Unis.

C'est sur cet aspect que j'aimerais conclure. La présenceabsence de l'Amérique doit en effet permettre de dépasser cette logique du consensus que la série fait prévaloir. Finalement, aussi nuancée semble-t-elle en apparence, la représentation du politique conduit à un idéalisme dont il faut noter les limites. Celles-ci sont à chercher justement dans le contre-champ de la décision politique. Voir de trop près l'Amérique, c'est dévoiler une souffrance, un mal-être liés aux choix opérés par les personnages. Sorkin et Wells préfèrent ostensiblement nous montrer le désespoir et le fardeau des responsabilités sur le visage du président et de ses conseillers. Conscients des conséquences néfastes de décisions qu'ils sont parfois sommés de prendre, soumis à des dilemmes cornéliens, ils n'ont plus qu'à vivre avec ce poids. Mais où sont les citoyens victimes de ces choix ? Comment dévoiler l'irreprésentable? Deux exemples : Bartlet envoie des hommes réparer une centrale nucléaire suite à un accident (7.12). Pendant ce temps, la population est touchée par les radiations et se presse sur les routes; ces faits seront narrés par le discours des politiques ou par le truchement des images télévisées. Autre cas : Josh Lyman, conseiller du président, signe des accords commerciaux (5.19). Dans son camp comme chez les républicains, on le félicite. Mais le personnage découvre que cette réussite politique se soldera par des délocalisations massives en Inde.

Dans les deux situations, on voit les limites d'une série à dispositif dans laquelle il est impossible qu'une scène se déroule en marge du personnel politique. Par ailleurs, montrer un Américain perdant son emploi, ou un chômeur ne trouvant pas de travail, n'aura pas la même incidence sur les téléspectateurs citoyens que de s'arrêter sur le regard pensif d'un conseiller remportant une victoire à la Pyrrhus. Enfin, en ne voyant rien des corps irradiés suite à l'accident nucléaire, on évite certes le spectaculaire et un pathos facile, mais on leur substitue une pudeur ostentatoire, où le visage du président se voit chargé d'incarner une région ravagée (voir figure 17). 


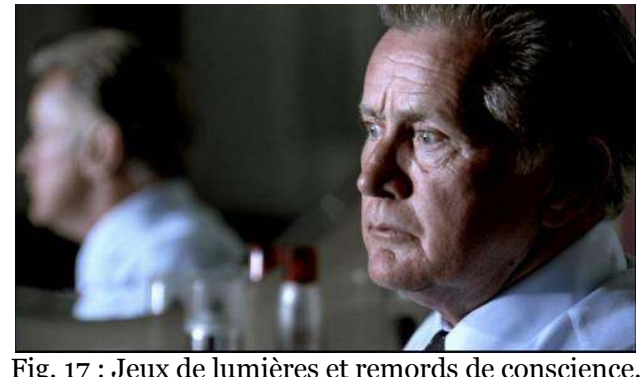

Visage marqué de Martin Sheen, symbole d'une certaine idée de l'Amérique, visage constamment travaillé par la lumière mais qui laisse dans l'ombre un territoire divers, un peuple divisé et souffrant.

\section{Bibliographie}

BIDAUD ANNE-Marie, Hollywood et le rêve américain; cinéma et idéologie, Paris, Masson, 1994.

Desbarats Carole, «À la Maison Blanche analysé par Carole Desbarats », cours du 15 avril 2011, www.forumdesimages.fr/fdi/LAcademie/A-voir-a-ecouter/Les-Cours-de-cinema-en-video, consulté le 5 août 2011.

THORET JEAN-Baptiste, Le cinéma américain des années 70, Paris, Cahiers du Cinéma, 2006. 